[2] Moreau R, Jalan R, Gines P, Pavesi M, Angeli P, Cordoba J, et al. Acute-onchronic liver failure is a distinct syndrome that develops in patients with acute decompensation of cirrhosis. Gastroenterology 2013;144:1426-1437.

[3] Kerbert AJ, Weil D, Verspaget HW, Moréno JP, van Hoek B, Cervoni JP, et al. Copeptinis an independentprognostic factor for transplant-free survival in cirrhosis. Liver Int 2016;36:530-537.

[4] Ruf AE, Villamil FG. C-reactiveprotein and model for end-stage liverdiseasescore: Have wefound the fifthelement? Liver Transpl 2015;21:713-715.

[5] Durand F, Buyse S, Francoz C, Laouénan C, Bruno O, Belghiti J, et al. Prognostic value of muscle atrophy incirrhosisusingpsoas muscle thickness on computedtomography. J Hepatol 2014;60:1151-1157.

[6] Cervoni JP, Thévenot T, Weil D, Muel E, Barbot O, Sheppard F, et al. Creactiveproteinpredicts short-termmortality in patients withcirrhosis. J Hepatol 2012;56:1299-1304.

[7] Cervoni JP, Amorós À, Bañares R, Luis Montero J, Soriano G, Weil D, et al. Prognostic value of C-reactiveprotein in cirrhosis:external validation from the CANONIC cohort. Eur J Gastroenterol Hepatol 2016;28:1028-1034.

[8] Di Martino V, Coutris C, Cervoni JP, Dritsas S, Weil D, Richou C, et al. Prognostic value of C-reactiveproteinlevels in patients withcirrhosis. Liver Transpl 2015;21:753-760.

[9] Li Xue, Chan Tung O, Myers Valerie, Chowdhury Ibrul, Zhang Xue-Qian, Song Jianliang, et al. Controlled and cardiac-restricted overexpression of the arginine vasopressin $\mathrm{V} 1 \mathrm{~A}$ receptor causes reversible left ventricular

\section{JOURNAL OF HEPATOLOGY}

dysfunction through $\mathrm{G} \alpha_{\mathrm{q}}$-mediated cell signaling. Circulation 2011;124: $572-581$.

[10] Krag A, Bendtsen F, Henriksen JH, Møller S. Low cardiac output predicts development of hepatorenal syndrome and survival in patients with cirrhosis and ascites. Gut 2010;59:105-110.

Thierry Thevenot

Delphine Weil

Jean-Paul Cervoni ${ }^{1, *}$

Federico G. Villamil ${ }^{2}$

Vincent Di Martino ${ }^{1}$

${ }^{1}$ Service d'Hépatologie et de Soins Intensifs Digestifs,

Hôpital Jean Minjoz, 25030 Besançon cedex, France

${ }^{2}$ Liver Transplant Unit, British Hospital, Perdriel 74, CABA (AEB1280),

Buenos Aires, Argentina

* Corresponding author. Address: Service d'Hépatologie et de Soins Intensifs Digestifs, Hôpital Jean Minjoz,

25000 Besançon, France.

Tel.: +33 3816684 21; fax: +33 381668418

E-mail address: jpcervoni@chu-besancon.fr

\title{
Reply to: "Are we still searching for the fifth element of MELD?"
}

To the Editor:

We would like to thank the authors for their interest in our study. Overall, our study was not aimed at looking for a fifth element of MELD score but investigating the role of copeptin as biomarker of circulatory dysfunction, disease progression, and prognosis in cirrhosis. The main finding our study was that copeptin was not only an independent predictive factor of mortality but also of acute decompensation during a 3-month follow-up period. Moreover, the study showed that copeptin, as a good surrogate marker of vasopressin (AVP), likely reflected the progressive impairment of circulatory function of patients with decompensated cirrhosis [1]. The main results of our study were internally and externally validated, which supports the robustness of the findings. Indeed, our results are in keeping with a previous study from the authors of this letter in which for the first time they showed that copeptin levels increased with liver disease severity and that were an independent predictive factor of 1-year mortality [2]. Therefore, considering their previous results and the fact that our results were externally validated, it appears that the prognostic value of copeptin is reliable. We agree with the authors that ideally, it would have been better to use a larger sample size for external validation, however, this population was selected using a 1:2 ration from the population of the study, and rather than being single-centre it includes patients from different European hospitals. Finally, of the 120 patients from the validation series, 25 died (20\%) and 11 were transplanted (9\%) during the 3-month followup period, which is a similar proportion of outcomes to that of patients from the study cohort [1].

While in our study, the prognostic model included copeptin together with MELD and leukocyte count, in the previous study from these authors the model included copeptin, MELD score and CRP. As indicated by the authors, CRP has already been shown as a marker of prognosis in cirrhosis [3-5]. Unfortunately, we did not measure CRP in our study so it could not be compared to the prognostic value of copeptin. Nevertheless, we agree with the authors that it would have been an interesting analysis. However, it is probable that copeptin and CRP (or leukocyte count in our study) reflect different aspects related to the prognosis of patients with cirrhosis. Results of our study suggest that copeptin reflects circulatory dysfunction of patients with cirrhosis. However, it is important to note that both CRP and leukocyte count are markers of inflammation. Moreover, in recent years there has been increasing evidence indicating that a systemic inflammatory reaction may play an important role in the progression of cirrhosis, development of complications and prognosis together with the impairment of circulatory function [6]. Therefore, it appears that prognostic models of both studies included three variables representing the most relevant factors affecting prognosis in cirrhosis (1) MELD score, (2) copeptin as a marker of circulatory dysfunction and (3) a marker or inflammation (either CRP or leukocytes). Whether copeptin has better accuracy than CRP to predict prognosis should be investigated in future studies.

Finally, the authors of this letter raise three important points, which we agree are of great interest, although unfortunately are not addressed in our paper as they were not the aim of our study: (1) serial measurements of copeptin, (2) the usefulness of copeptin in patients in the waiting list for liver transplantation and (3) the relationship of copeptin levels and ACLF. Therefore, we believe that it would be worth investigating these ideas in further studies. 


\section{Letters to the Editor}

\section{Financial support}

The work discussed in this paper has been funded by grants FIS PI12/00330 and FIS08/0126 integrated in the Plan Nacional $\mathrm{I}+\mathrm{D}+\mathrm{I}$ and co-funded by ISCIII. CIBEREHD is funded by the Instituto de Salud Carlos III. Additional support was obtained from Thermofisher Scientific Inc (Hennigsdorf, Germany). Part of this work (validation series) has been supported by the EASL-CLIF Consortium and European Foundation for the Study of Chronic Liver Failure (EF-CLIF) which is supported by an unrestricted grant from Grifols. Pere Ginès is recipient of an Institució Catalana de Recerca i Estudis Avançats (ICREA) Academia Award.

\section{Conflict of interest}

Pere Ginès received financial support from Thermofisher Scientific Inc and research grants from Grifols and Sequana Medical. The other authors have nothing to disclose.

\section{Authors' contributions}

The authors jointly drafted and critically revised the manuscript and intellectual content on behalf of the co-authors of the original study.

\section{References}

[1] Solà E, Kerbert JC, Verspaget HW, Moreira R, Pose E, Ruiz P, et al. Plasma copeptin as biomarker of disease progression and prognosis in cirrhosis. Hepatol 2016;65:914-920.

[2] Moreno JP, Grandclement E, Monnet E, Clerc B, Agin A, Cervoni JP, et al. Plasma copeptin, a possible prognostic marker in cirrhosis. Liver Int 2013;33:843-851.

[3] Cervoni JP, Thévenot T, Weil D, Muel E, Barbot O, Sheppard F, et al. C-reactive protein predicts short-term mortality in patients with cirrhosis. J Hepatol 2012;56:1299-1304.

[4] Cervoni JP, Amorós À, Bañares R, Luis Montero J, Soriano G, Weil D, et al. Prognostic value of C-reactive protein in cirrhosis: external validation from the CANONIC cohort. Eur J Gastroenterol Hepatol 2016;28:1028-1034.

[5] Di Martino V, Coutris C, Cervoni JP, Dritsas S, Weil D, Richou C, et al. Prognostic value of C-reactive protein levels in patients with cirrhosis. Liver Transpl 2015;21:753-760.

[6] Bernardi M, Moreau R, Angeli P, Schanbl B, Arroyo V. Mechanisms of decompensation and organ failure in cirrhosis: From peripheral arterial vasodilation to systemic inflammation hypothesis. J Hepatol 2015;63:1272-1284.

Elsa Solà ${ }^{1,2,3, *}$
Annarein Kerbert ${ }^{4}$
Minneke Coenraad ${ }^{4}$
Pere Ginès ${ }^{1,2,3}$
Liver Unit, Hospital Clínic, University of Barcelona, Barcelona, Spain
${ }^{2}$ Institut d'Investigacions Biomèdiques August Pi i Sunyer
(IDIBAPS), Spain
${ }^{3}$ Centro de Investigación Biomédica en Red de Enfermedades
Hepáticas y Digestivas (CIBEREHD), Spain
${ }^{4}$ Department of Gastroenterology-Hepatology,
* Leiden University Medical Center, Leiden, The Netherlands
University of Barcelona, Villarroel 170, 08036 Barcelona, Spain.
Tel.: +34 932271713.
E-mail address: esola@clinic.cat

To the Editor:

We read with interest the paper by Potze et al. [1], in which they investigated non-alcoholic-fatty-liver disease (NAFLD) and concluded that hemostasis (i.e., platelets, coagulation and fibrinolysis) is rebalanced. We recently reported results on the same topic, but only for coagulation [2]. Our study aimed at ensuring fair comparability of results (patients vs. controls). The numbers of controls were nearly two times higher than those of patients. Blood samples were collected and tested within the same hospital in a defined period. To minimize analytical variability, samples from appropriate numbers of patients and controls were analyzed (with/without thrombomodulin) within the same working-sessions. Based on our results, we concluded that NAFLD plasmas displayed biochemical signs of procoagulant imbalance as shown by increased endogenous thrombin potential (ETP) ratio (with/without thrombomodulin). The procoagulant imbalance progressed from steatosis (the least severe form of NAFLD) to metabolic cirrhosis (the most severe). Metabolic cirrhosis had ETP ratios of a similar magnitude to those for alcoholic/viral cirrhosis. These conclusions were also supported (in the same study) by the results of another thrombin generation assay based on the presence/absence of Protac (a non-physiologic protein $C$ $[\mathrm{PC}]$ activator). We also found increased factor VIII (FVIII) (one of the most potent procoagulants), and decreased PC (one of the most potent anticoagulants) in NAFLD patients. Results of the two thrombin generation assays correlated with FVIII, PC and FVIII to PC ratio. Finally, patients with ETP ratios higher than the median control value had odds ratios higher than 1.0 for metabolic syndrome, liver fibrosis, steatosis, lobular inflammation, and NAFLD activity score, all considered typical features of NAFLD.

Results and conclusions are in line with the notion that NAFLD patients have increased cardiovascular risk and liver fibrosis as shown by clinical and epidemiological observations.

Potze et al., however, reached different conclusions: coagulation in NAFLD is rebalanced and there are no signs of procoagulant imbalance in spite of elevated FVIII and von Willebrand factor (another index of procoagulant imbalance [3]), and 